\section{A NOVEL HUMANIZED MURINE MODEL TO IDENTIFY NEOANTIGEN-SPECIFIC T CELLS IN PEDIATRIC ACUTE MEGAKARYOBLASTIC LEUKEMIA}

${ }^{1}$ Elizabeth Garfinkle*, ${ }^{2}$ Anthony Zamora, ${ }^{1}$ Jeremy Crawford, ${ }^{1}$ Paul Thomas, ${ }^{1}$ Tanja Gruber. ${ }^{1}$ St. Jude Children's Research Hospital, San Mateo, CA, USA; ${ }^{2}$ Medical College of Wisconsin, Milwaukee, WI, USA

Background CBFA2T3-GLIS2 is a chimeric transcription factor in $18.4 \%$ of pediatric patients with non-Down syndrome acute megakaryoblastic leukemia (non-DS-AMKL). It remains one of the poorest prognostic subtypes amongst pediatric acute myeloid leukemia patients with event free and overall survival probabilities of $8 \%$ and $14 \%$ respectively, despite intensive multi-agent chemotherapy and stem cell transplant treatment modalities. ${ }^{1}$ Adoptive cell therapies targeting the CBFA2T3GLIS2 fusion may provide additional treatment options for AMKL patients. We hypothesize CBFA2T3-GLIS2 fusion junction specific neoantigen(s) are presented by surface HLA class I expression and elicit functional immune responses by CD8+ T cells.

Methods To interrogate this hypothesis in silico, we used NetMHCcons to predict neoantigens spanning the fusion junction. $^{2}$ To test our hypothesis in vivo, we established a small cohort (total $\mathrm{N}=12$ ) of immunodeficient NSG-SGM3 mice engrafted with patient derived xenograft (PDX) cells from a CBFA2T3-GLIS2 positive AMKL patient for 20 days followed by the introduction of PBMCs from a healthy, unrelated donor with a 6/6 HLA class I match to the PDX cells for an additional 10, 15, or 20 days (figure 1). ${ }^{3}$ Leukemia and $\mathrm{T}$ cell phenotype was characterized by flow cytometry. Levels of soluble analytes were measured in the serum by cytometric bead array. The TCR repertoire and transcriptional profile of the CD8+ $\mathrm{T}$ cells was determined through paired gene expression and 5' single cell $\mathrm{V}(\mathrm{D}) \mathrm{J}$ sequencing using $10 \mathrm{X}$ Genomics.

Results NetMHCcons identified two neoantigens spanning the fusion junction with intermediate binding affinities (IC50) of 304.37 and 384.09 nM. Flow cytometry analysis at Day 40 (figure 1) showed dramatic reduction of leukemic blasts (huCD45lo, huCD34+) when exposed to PBMCs compared to Leukemia Only control (1.48\% and 93.7\%, respectively). Circulating human Granzyme A was elevated at Day 40 in the PDX mice with PBMCs $(9665.4 \mathrm{pg} / \mathrm{ml})$ compared to the

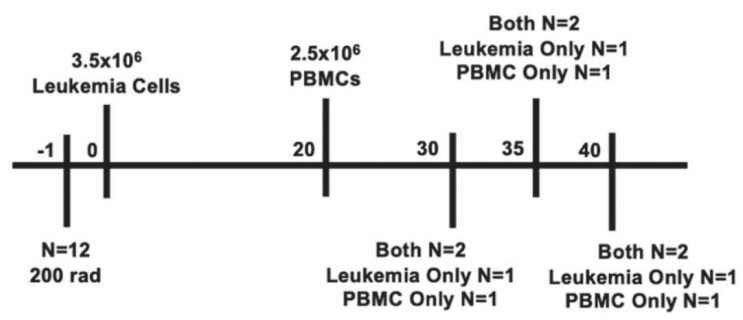

Abstract 737 Figure 1 Establishment of novel humanized PDX murine model.

12 immunodeficient NSG-SGM3 mice were irradiated with 200 rads the day before intravenous injection of patient-derived CBFA2T3-GLIS2 positive AMKL cells. After 20 days of engraftment, mice were intravenously injected with 6/6 HLA class I matched PBMCs. 2 mice with both leukemia cells and PBMCs, 1 mouse with only leukemia cells, and 1 mouse with only PBMCs were collected at Days 30, 35, and 40. Human cells were recovered from the murine model by depleting mCD45 + cells from the bone marrow and spleen using magneticactivated cell sorting

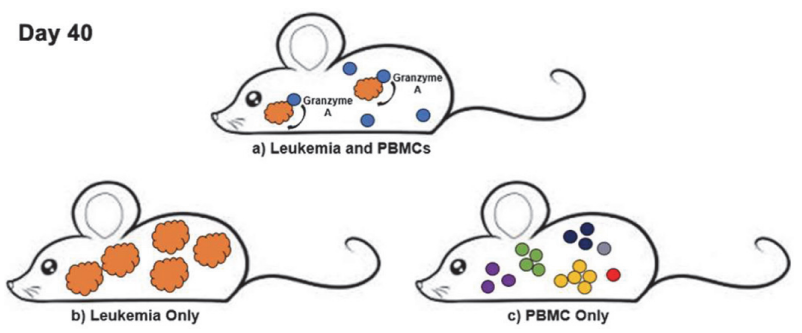

Abstract 737 Figure 2 Leukemia cells targeted by clonal CD8+ T cells.

At Day 40, our humanized murine model showed reduced leukemia burden when exposed to PBMCs (a) compared to a Leukemia Only control (b). This response is mediated by the release of Granzyme $A$ from a subset of uniform, clonally expanded leukemia-specific CD8+ T cells (a) that are not present in the highly diverse expanded TCR repertoire of the PBMC Only control (c). Colored circles represent CD8+ T cells with different TCRs. Orange symbol represents leukemia cells

PBMC Only control $(789.7 \mathrm{pg} / \mathrm{ml})$, indicating a potential leukemia-directed cytokine lymphocyte response. TCR repertoire sequencing of CD8 $+\mathrm{T}$ cells from Day 40 revealed a decrease in alpha beta TCR diversity when exposed to PDX cells $\left(10^{4}\right.$ TCRdiv) compared to the PBMC Only control (10 ${ }^{7}$ TCRdiv), consistent with a clonal expansion of neoantigen-specific TCRs $^{4}$ (figure 2). Specificity of the TCRs for the putative neoantigens was assessed by cloning top expanded TCRs to measure tetramer binding and functional response. ${ }^{5}$

Conclusions Our novel murine model demonstrates a potential neoantigen-directed $\mathrm{CD} 8+\mathrm{T}$ cell response against patient AMKL cells, highlighting the importance of adoptive cell therapy to improve patient outcomes.

Acknowledgements We would like to thank Scott Perry in the Flow Cytometry and Cell Sorting Shared Resource (SJCRH) for his help with flow cytometry and the Animal Resource Center (SJCRH) staff for the care and housing of the murine model described here. This work is supported by the American Lebanese Syrian Associated Charities (ALSAC) (T.A.G., P. G.T.), R01 AI136514 (P.G.T.), and the Mark Foundation ASPIRE award (P.G.T.).

Ethics Approval The vertebrate animal studies described here were approved by the Institutional Animal Care and Use Committee (IACUC); animal welfare assurance number A332501. The patient derived xenograft leukemia cells and healthy donor PBMCs used in the studies described here are exempt from requiring Institutional Review Board (IRB) approval because we receive no personal identifying information about the patient/donor from which the xenograft cells/PBMCs are derived.

\section{REFERENCES}

1. de Rooij J, Branstetter C, Ma J, Li Y, et al. Pediatric non-Down syndrome acute megakaryoblastic leukemia is characterized by distinct genomic subsets with varying outcomes. Nat. Genet 2017; 49:451-456.

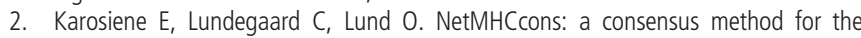
major histocompatibility complex class I predictions. Immunogenetics 2012; 64:269-281.

3. Roth $M$, Harui A. Human tumor infiltrating lymphocytes cooperatively regulate prostate tumor growth in a humanized mouse model. J Immunother Cancer. 2015; 10:3-11.

4. Dash P, Fiore-Gartland A, Wang $G$, et al. Quantifiable predictive features define epitope-specific T cell receptor repertoires. Nature 2017; 547:89-93.

5. Zamora A, Crawford J, Allen E, Kuo X, et al. Pediatric patients with acute lymphoblastic leukemia generate abundant and functional neoantigen-specific CD8+ T cell responses. Sci Trans/ Med 2019; 11:eaat8549.

http://dx.doi.org/10.1136/jitc-2020-SITC2020.0737 